\title{
Cohomology Algebras of Blocks of Finite Groups and Brauer Correspondence II *
}

\author{
Hiroki Sasaki \\ School of General Education, Shinshu University, Matsumoto, 390-8621, Japan \\ email: sasaki@math.shinshu-u.ac.jp
}

\begin{abstract}
Let $k$ be an algebraically closed field of characteristic $p$. We shall discuss the cohomology algebras of a block ideal $B$ of the group algebra $k G$ of a finite group $G$ and a block ideal $C$ of the block ideal of $k H$ of a subgroup $H$ of $G$ which are in Brauer correspondence and have a common defect group, continuing [4]. We shall define a $(B, C)$-bimodule $L$. The $k$-dual $L^{*}$ induces the transfer map between the Hochschild cohomology algebras of $B$ and $C$, which restricts to the inclusion map of the cohomology algebras of $B$ into that of $C$ under some condition. Moreover the module $L$ induces a kind of refinement of Green correspondence between indecomposable modules lying in the blocks $B$ and $C$; the block varieties of modules lying in $B$ and $C$ which are in Green correspondence will also be discussed.
\end{abstract}

Keywords: finite group, block, source modules, Brauer correspondence, Green correspondence, Hochschild cohomology, block cohomology, block variety

Mathematics Subject Classification (2000): Primary 20C20

\section{Introduction}

Throughout this paper we let $k$ be an algebraically closed field of prime characteristic $p$.

Let $G$ be a finite group of order divisible by $p$. Proposition 2.3 of Kessar, Linckelmann and Robinson [5] says that the cohomology algebra $H^{*}(G, B)$ of a block ideal $B$ of the group algebra $k G$ is contained in the cohomology algebra $H^{*}(H, C)$ of a suitably taken block ideal $C$, which satisfies $C^{G}=B$, of a suitably chosen subgroup $H$ of $G$.

To understand such an inclusion via transfer map between the Hochschild cohomology algebras of the block ideals $B$ and $C$ we discussed in [4] under the following situation. Namely a block ideal $B$ of $k G$ has $D$ as a defect group; $H$ is a subgroup of $G$ and $C$ is a block ideal of $k H$ such that the Brauer correspondent $C^{G}$ is defined and $C^{G}=B$ and $D$ is also a defect group of $C$. We considered the $(C, B)$-bimodule $M=C B$ and gave a necessary and sufficient condition for $M$ to induce the transfer map from $H H^{*}(B)$ to $H H^{*}(C)$ which restricts to the inclusion map of $H^{*}(G, B)$ into $H^{*}(H, C)$.

In this paper we shall discuss under the following situation:

* This work is supported by Grant-in-Aid for Scientific Research (C) (17540032), Japan Society for the Promotion of Science. 
Situation (BC). Let $B$ be a block ideal of $k G$ with a defect group $D$; let $H$ be a subgroup of $G$ containing $D C_{G}(D)$ and $C$ a block ideal of $k H$ such that $C^{G}=B$. Assume that $C$ has $D$ as a defect group.

We shall denote by $G^{\text {op }}$ the opposite group of the group $G$ and consider the group algebra $k G$ as a $k\left[G \times G^{\mathrm{op}}\right]$-module through $(x, y) \alpha=x \alpha y$ for $x, y \in G$ and $\alpha \in k G$. The stabilizer of $1 \in G$ under the action of $G \times G^{\text {op }}$ on $G$ is the diagonal set $\Delta G=\left\{\left(g, g^{-1}\right) \mid g \in G\right\}$; hence we have a $k[G \times$ $\left.G^{\mathrm{op}}\right]$-isomorphism $k G \simeq k\left[G \times G^{\mathrm{op}}\right] \otimes_{\Delta G} k$.

Definition 1 Under Situation (BC), because the block ideal $C$ has, as an indecomposable $k\left[H \times H^{\mathrm{op}}\right]$-module, $\Delta D$ as a vertex and $N_{G \times H^{\mathrm{op}}}(\Delta D)=$ $\Delta N_{H}(D)\left(C_{G}(D) \times 1\right) \leqslant H \times H^{\text {op }}$, the Green correspondent of $C$ to $G \times H^{\text {op }}$ is defined, which turns out to be a $(B, C)$-bimodule; we denote it by $L(B, C)$.

The module $L(B, C)$ will play crucial role in this paper, depending on the following fact, which will be proved in Section 2.

Theorem 1.1 Under Situation (BC) let $L=L(B, C)$. The relatively $L$-projective element $\pi_{L} \in Z(B)$ and the relatively $L^{*}$-projective element $\pi_{L^{*}} \in Z(C)$ are both invertible. In particular, A being an arbitrary symmetric algebra over $k$ of finite dimension, every finitely generated $(B, A)$-module is relatively L-projective and every finitely generated $(C, A)$-module is relatively $L^{*}$-projective.

For the theory of projectivity relative to bimodules over symmetric algebras, see Section 5 Appendix, where we shall quote some definitions and results from Broué's lecture notes [3] for the convenience of the readers. We shall also state some facts which we shall use frequently.

Here we fix a symbol. For a subgroup $S$ of a finite group $T$ and a $k T$ module $W$ we shall write ${ }_{S} W$ for the restriction of $W$ to $k S$.

Following Alperin, Linckelmann and Rouquier [1], we recall the definition of source modules of block ideals.

Definition 2 ([1, Definition 2]) Since, as an indecomposable $k\left[G \times G^{\mathrm{op}}\right]$ module, $B$ has $\Delta D$ as a vertex and $G \times D^{\text {op }} \geqslant \Delta D$, there exists an indecomposable direct summand $X$ of the $k\left[G \times D^{\text {op }}\right]$-module ${ }_{G \times D^{\text {op }}} B$ having $\Delta D$ as a vertex. The $k\left[G \times D^{\text {op }}\right]$-module $X$ is called a source module of the block $B$. The source module $X$ is written as $k G i$, where $i$ is a source idempotent. If $X$ and $X^{\prime}$ are source modules of $B$, then they are conjugate under $N_{G}(D)$, namely $X \simeq X^{\prime} \otimes t$ as $k\left[G \times D^{\text {op }}\right]$-module for an element $t \in N_{G}(D)$.

We shall write $H^{*}(G, B ; X)$ for the block cohomology of $B$ with respect to the defect group $D$ and the source idempotent $i$ such that $X=k G i$. 
Under Situation (BC) we can take a source module $X$ of the block $B$ and a source module $Y$ of the block $C$ in order that $X$ and $Y$ are in the Green correspondence with respect to $\left(G \times D^{\mathrm{op}}, \Delta D, H \times D^{\mathrm{op}}\right)$. Then the $(B, C)$ bimodule $L=L(B, C)$ links the source modules $X$ and $Y$ in a similar way to induction and restriction of modules (Theorem 2.9). We should mention that the $(B, C)$-module $L(B, C)$ has already appeared in some works. In particular, in Alperin, Linckelmann and Rouquier [1] the case of $H=N_{G}\left(D, b_{D}\right)$, where $\left(D, b_{D}\right)$ is a Sylow $B$-subpair, was treated. Theorem 5 in [1] corresponds to our Theorem 2.9; our proof of Theorem 2.9 is partly due to the argument of the proof of [1, Theorem 5].

One of our ingredients to prove Theorem 1.2 below is that the bimodule $L$ is splendid with respect to $X$ and $Y$, namely $L$ is a direct summand of the tensor product $X \otimes_{k D} Y^{*}$ (Theorem 2.10). Another important property is that the relatively projective elements associated with tensor products of the bimodules $L, X$ and $Y$ and their duals, including such as $X^{*} \otimes_{B} L \otimes_{C} Y$, are all invertible (Theorem 2.11).

The following is one of our main theorems.

Theorem 1.2 Let $B$ be a block ideal of $k G$ and $D \leqslant G$ a defect group of $B$. Assume that a subgroup $H$ of $G$ containing $D C_{G}(D)$ normalizes a subgroup $Q$ of $D$ and contains $Q C_{G}(Q)$. Let $\left(D, b_{D}\right)$ be a Sylow B-subpair and let $\left(Q, b_{Q}\right) \leqslant\left(D, b_{D}\right)$. Let $C$ be a unique block ideal of $k H$ covering the block ideal $b_{Q}$ of $k Q C_{G}(Q)$. Then $C^{G}=B$ and $D$ is a defect group of $C$; hence $\left(D, b_{D}\right)$ is also a Sylow $C$-subpair.

Let $j$ be a source idempotent of $C$ such that $\operatorname{Br}_{D}(j) e_{D}=\operatorname{Br}_{D}(j)$, where $e_{D} \in k C_{G}(D)$ is the block idempotent of the block $b_{D}$; let $Y=k H j$. Let $X$ be a source module of $B$ which is the Green correspondent of $Y$ with respect to $\left(G \times D^{\mathrm{op}}, \Delta D, H \times D^{\mathrm{op}}\right)$. We let $L=L(B, C)$.

Then we have the following commutative diagram:

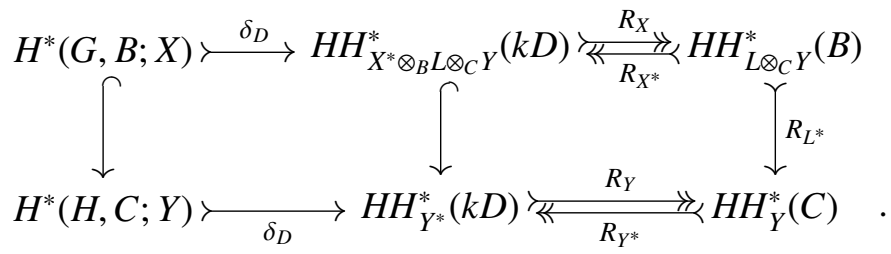

The theorem above will be proved in Section 3 .

In Section 4 we shall discuss block varieties of modules which are in Green correspondence.

If the cohomology algebra $H^{*}(G, B ; X)$ is contained in the cohomology algebra $H^{*}(H, C ; Y)$, then Kawai and Sasaki [4, Theorem 1.3 (i)] says that the inclusion map $\iota: H^{*}(G, B ; X) \hookrightarrow H^{*}(H, C ; Y)$ induces a surjective map $\iota^{*}: V_{H, C} \rightarrow V_{G, B}$ of varieties; this generalizes to varieties of modules as follows. 
Theorem 1.3 Under Situation (BC) let $L=L(B, C)$. Let $X$ and $Y$ be source modules of the blocks $B$ and $C$, respectively. Assume that $X$ and $Y$ are in the Green correspondence with respect to $\left(G \times D^{\mathrm{op}}, \Delta D, H \times D^{\mathrm{op}}\right)$. We let moreover $P \leqslant D$ and assume that the subgroup $H$ contains $N_{G}(P)$.

Assume that $H^{*}(G, B ; X) \subset H^{*}(H, C ; Y)$.

(i) Assume that an indecomposable B-module $U$ has an $X$-vertex belonging to $\mathscr{A}(G, P, H)$. Then the Green correspondent $V$ of $U$ to $H$ with respect to $(G, P, H)$ lies in the block $C$ and the following holds:

$$
V_{G, B}(U)=\iota^{*} V_{H, C}(V) .
$$

(ii) Assume that an indecomposable $C$-module $V$ has a $Y$-vertex belonging to $\mathscr{A}(G, P, H)$. Then the Green correspondent $U$ of $V$ to $G$ with respect to $(G, P, H)$ lies in the block $B$ and the following holds:

$$
V_{G, B}(U)=\iota^{*} V_{H, C}(V) .
$$

Benson and Linckelmann [2] showed that the block variety of an indecomposable $k G$-module lying in a block is determined by a particular vertex and a source that are compatible with a source modules of the block ideal. We have to state more precisely.

Definition 3 ([2, Proposition 2.5]) Let $X$ be a source module of a block ideal $B$. Let $U$ be an indecomposable $k G$-module lying in $B$. There exists a vertex $Q$ of $U$ such that

$$
Q \leqslant D, \quad U \mid X \otimes_{k Q} X^{*} \otimes_{B} U .
$$

We would like to call such a vertex $Q$ of $U$ an $X$-vertex. For an $X$-vertex $Q$ of $U$ we can take a $Q$-source $S$ such that

$$
U\left|X \otimes_{k Q} S, \quad S\right|{ }_{Q} X^{*} \otimes_{B} U .
$$

We would like to call such a source a $(Q, X)$-source.

[2, Theorem 1.1] says that the block variety $V_{G, B}(U)$ in the block cohomology $H^{*}(G, B ; X)$ is the pull back of the variety $V_{Q}(S)$ of $S$, where $Q$ is an $X$ vertex and $S$ is a $(Q, X)$-source of $U$. Depending on this fact, [2, Corollary 1.4] says that the Green correspondents have the same block varieties for a particular case. We would like to understand this phenomenon from more general context.

Under Situation (BC) and the assumption that the source modules $X$ and $Y$ of the block ideals $B$ and $C$ are in Green correspondence, our bimodule $L=L(B, C)$ gives rise to a kind of refinement of Green correspondence (Proposition 4.1). The theorem above depends on the fact that Green correspondents have a common vertex and a common source compatible with 
source modules of the block ideals; see Proposition 4.4, which will be proved by using the bimodule $L$.

\section{Brauer Correspondence}

First of all we state a couple of lemmas concerning source modules of block ideals. Let $X=k G i$ be a source module of the block $B$. Let $e_{D} \in k C_{G}(D)$ be the block idempotent such that $\operatorname{Br}_{D}(i) e_{D} \neq 0$. The following are well known.

Lemma 2.1 The $k\left[D C_{G}(D) \times D^{\mathrm{op}}\right]$-module $k\left[D C_{G}(D)\right] \mathrm{Br}_{D}($ i) is a source module of the block ideal $k\left[D C_{G}(D)\right] e_{D}$.

Since $k G i$ is a direct summand of $k G \operatorname{Br}_{D}(i)$ as $k\left[G \times D^{\mathrm{op}}\right]$-modules, we have

Lemma 2.2 The source module $X=k G i$ is the Green correspondent of the source module $k\left[D C_{G}(D)\right] \mathrm{Br}_{D}(i)$ of the block $k\left[D C_{G}(D)\right] e_{D}$ with respect to $\left(G \times D^{\mathrm{op}}, \Delta D, D C_{G}(D) \times D^{\mathrm{op}}\right)$.

In the rest of this section we argue under Situation (BC) and we let $L=$ $L(B, C)$.

Since $N_{G \times D^{\text {op }}}(\Delta D)=\Delta D\left(C_{G}(D) \times 1\right) \leqslant H \times D^{\text {op }}$, the Green correspondence between the indecomposable $k\left[G \times D^{\text {op }}\right]$-modules with vertices in $\mathscr{A}(G \times$ $\left.D^{\mathrm{op}}, \Delta D, H \times D^{\mathrm{op}}\right)$ and the indecomposable $k\left[H \times D^{\mathrm{op}}\right]$-modules with vertices in $\mathscr{A}\left(G \times D^{\mathrm{op}}, \Delta D, H \times D^{\mathrm{op}}\right)$ makes sense.

Proposition 2.3 Let $Y$ be a source module of the block ideal $C$ and let $X$ be the Green correspondent of $Y$ with respect to $\left(G \times D^{\mathrm{op}}, \Delta D, H \times D^{\mathrm{op}}\right)$. Then the following hold.

(i) The $k\left[G \times D^{\mathrm{op}}\right]$-module $X$ is a source module of the block $B=C^{G}$.

(ii) The Brauer constructions $X(D)$ and $Y(D)$ are isomorphic as $k C_{G}(D)$ modules.

Hence if $\left(D, b_{D}\right)$ is a Sylow $C$-subpair such that $b_{D} Y(D) \neq 0$, then $\left(D, b_{D}\right)$ is a Sylow $B$-subpair and $b_{D} X(D) \neq 0$.

Proof. (i) Because

$$
H \times D^{\text {op }} B={ }_{H \times D^{\text {op }}}\left(H \times H^{\text {op }} B\right)={ }_{H \times D^{\text {op }}}\left(C \oplus C^{\prime}\right)=Y \oplus \cdots
$$

and $H \times D^{\text {op }} B=H \times D^{\text {op }}\left(G \times D^{\text {op } B}\right)$, there exists an indecomposable direct summand $X^{\prime}$ of $G \times D^{\text {op }} B$ such that $Y \mid H \times D^{\text {op }} X^{\prime}$. Theorem of Burry, Carlson and Puig (Nagao and Tsushima [8, Theorem 4.4.6]) implies that $X^{\prime}$ is the Green 


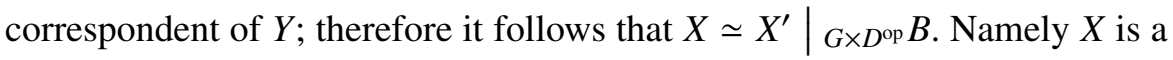
source module of $B$.

(ii) Since $B=C^{G}=\left(b_{D}{ }^{H}\right)^{G}=b_{D}{ }^{G}$, we see that $\left(D, b_{D}\right)$ is a Sylow $B$ subpair. Because $H \times D^{\mathrm{op}} X \simeq Y \oplus Y_{0}$, where $Y_{0}$ is projective relative to $\mathscr{Y}(G \times$ $\left.D^{\mathrm{op}}, \Delta D, H \times D^{\mathrm{op}}\right)$, the $k[\Delta D]$-module ${ }_{\Delta D} Y_{0}$ is projective relative to $\{\Delta Q$ | $Q<D\}$. Therefore we see that $Y_{0}(D)=0$, hence $X(D) \simeq Y(D)$ as $k C_{G}(D)$ modules.

Proposition 2.4 Let $X$ be a source module of $B$ and $Y$ a source module of $C$. If the Brauer constructions $X(D)$ and $Y(D)$ are isomorphic as $k C_{G}(D)$ modules, then the modules $X$ and $Y$ are in the Green correspondence with respect to $\left(G \times D^{\mathrm{op}}, \Delta D, H \times D^{\mathrm{op}}\right)$.

Proof. We write $X=k G i$ and $Y=k H j$, where $i$ and $j$ are source idempotents of $B$ and $C$, respectively. Let $\widehat{X}(D)=k\left[D C_{G}(D)\right] \operatorname{Br}_{D}(i)$ and $\widehat{Y}(D)=$ $k\left[D C_{H}(D)\right] \mathrm{Br}_{D}(j)$. Then a $k C_{G}(D)$-isomorphism from $X(D)$ to $Y(D)$ lifts to a $k\left[D C_{G}(D)\right]$-isomorphism from $\widehat{X}(D)$ to $\widehat{Y}(D)$, which is also an isomorphism of $k\left[D C_{G}(D) \times D^{\mathrm{op}}\right]$-modules. We see from Lemma 2.2 that

- the source module $X$ of $B$ is the Green correspondent of $\widehat{X}(D)$ with respect to $\left(G \times D^{\mathrm{op}}, \Delta D, D C_{G}(D) \times D^{\mathrm{op}}\right)$,

- the source module $Y$ of $C$ is the Green correspondent of $\widehat{Y}(D)$ with respect to $\left(H \times D^{\mathrm{op}}, \Delta D, D C_{G}(D) \times D^{\mathrm{op}}\right)$.

Thus the modules $X$ and $Y$ correspond under the Green correspondence with respect to $\left(G \times D^{\mathrm{op}}, \Delta D, H \times D^{\mathrm{op}}\right)$.

Lemma 2.5 The following holds.

$$
B \otimes_{k H} C \equiv L \oplus O\left(\mathscr{X}\left(G \times H^{\mathrm{op}}, \Delta D, H \times H^{\mathrm{op}}\right)\right) .
$$

In particular, the module $L$ is a $(B, C)$-bimodule.

Proof. Put $M=B \otimes_{k H} C$. We first show that $L$ is a direct summand of $M$ as $k\left[G \times H^{\text {op }}\right]$-modules. Notice that the block ideal $C$ is a direct summand of $H \times H^{\circ \mathrm{op}} M$. Let $M=L_{1} \oplus \cdots \oplus L_{n}$ be a direct sum decomposition into indecomposable $k\left[G \times H^{\mathrm{op}}\right]$-modules. Then the block ideal $C$ is a direct summand of $H \times H^{\circ \mathrm{op}} L_{i}$ for some $L_{i}$. Theorem of Burry, Carlson and Puig says that $L_{i}$ is the Green correspondent of $C$. Thus we obtain that $L \simeq L_{i}$ and that $L \mid M$. This together with the fact that $M \mid k\left[G \times H^{\mathrm{op}}\right] \otimes_{k\left[H \times H^{\mathrm{op}}\right]} C=$ $L \oplus O\left(\mathscr{X}\left(G \times H^{\mathrm{op}}, \Delta D, H \times H^{\mathrm{op}}\right)\right)$ gives rise to our assertion. 
Proposition 2.6 Since L is isomorphic with a direct summand of $G \times H^{\circ \mathrm{p}} B$, we can take a source module $X$ of the block ideal $B$ as a direct summand of $G \times D^{\mathrm{op}} L$. Then the Green correspondent $Y$ of $X$ to $H \times D^{\text {op }}$ with respect to $\left(G \times D^{\mathrm{op}}, \Delta D, H \times D^{\mathrm{op}}\right)$ is a source module of the block ideal $C$.

Proof. Let us write $H \times H^{\text {op }} L=C \oplus C_{0}$, where $C_{0} \equiv O\left(\mathscr{Y}\left(G \times H^{\text {op }}, \Delta D\right.\right.$, $\left.\left.H \times H^{\mathrm{op}}\right)\right)$. Then the $k\left[H \times H^{\mathrm{op}}\right]$-module $C_{0}$ has no indecomposable direct summands with vertex $\Delta D$; so does the $k\left[H \times D^{\text {op }}\right]$-module $H \times D^{\text {op }} C_{0}$.

The direct sum decomposition $H \times D^{\text {op }} X \mid H \times D^{\text {op }} L \simeq H \times D^{\text {op }} C \oplus H \times D^{\text {op }} C_{0}$ implies that the $k\left[H \times D^{\mathrm{op}}\right]$-module $Y$ is a direct summand of $H \times D^{\text {op }} C$; thus the Green correspondent $Y$ is a source module of the block $C$.

Before proving Theorem 1.1, we prepare the following lemma, which depends on the theory of projectivity relative to bimodules over symmetric algebras. A brief account of the theory is in Appendix.

Lemma 2.7 Let $B$ be a block ideal of $k G$ and $D$ a defect group of $B$. Let $H \leqslant G$ and $C$ a block ideal of $k H$. Assume that $D$ is a defect group of $C$. Suppose that an indecomposable $(B, C)$-bimodule $S$ has a trivial source and a vertex which is a proper subgroup of $\triangle D$. Then the relatively projective elements $\pi_{S} \in Z(B)$ and $\pi_{S^{*}} \in Z(C)$ are both nilpotent.

Proof. Let $\Delta Q, Q<D$, be a vertex of $S$. Because $S$ has a trivial source, it follows that

$$
S \mid k\left[G \times H^{\mathrm{op}}\right] \otimes_{k[\Delta Q]} k=k G \otimes_{k Q} k H .
$$

Namely, being $X={ }_{B} k G_{k Q}$ and $Y={ }_{k Q} k H_{C}$, we have that $S \mid X \otimes_{k Q} Y$. Then Theorem 5.4 implies that

$$
\begin{aligned}
\widehat{\pi}_{S} & \in \operatorname{Im}\left[{ }^{X} \operatorname{Tr}:{ }_{k Q}\left(X^{*}, X^{*}\right) \rightarrow{ }_{B}(B, B)\right], \\
\widehat{\pi}_{S^{*}} & \in \operatorname{Im}\left[{ }^{Y^{*}} \operatorname{Tr}:{ }_{k Q}(Y, Y) \rightarrow{ }_{C}(C, C)\right] .
\end{aligned}
$$

Thus we see that the relatively $S$-projective element $\pi_{S}=\widehat{\pi}_{S}(1) \in Z(B)$ belongs to the image of the relative trace map $\operatorname{tr}_{Q}^{G}: B^{Q} \rightarrow Z(B)$ and that the relatively $S^{*}$-projective element $\pi_{S^{*}}=\widehat{\pi}_{S^{*}}(1) \in Z(C)$ belongs to the image of the relative trace map $\operatorname{tr}_{Q}^{H}: C^{Q} \rightarrow Z(C)$. Because the blocks $B$ and $C$ have $D$ as a defect group and $Q<D$, we have that

$$
\operatorname{tr}_{Q}^{G}\left(B^{Q}\right) \subset J(Z(B)), \quad \operatorname{tr}_{Q}^{H}\left(C^{Q}\right) \subset J(Z(C))
$$

so that the elements $\pi_{S}$ and $\pi_{S^{*}}$ are nilpotent.

Proof of Theorem 1.1. Let $M=B \otimes_{k H} C$. Then, by Lemma 2.5, the $(B, C)$ bimodule $M$ decomposes as follows:

$$
M=L \oplus L_{0}, \quad L_{0} \equiv O\left(\mathscr{X}\left(G \times H^{\mathrm{op}}, \Delta D, H \times H^{\mathrm{op}}\right)\right) .
$$


We know from Kawai and Sasaki [4, Proposition 3.3 (b)] that the relatively projective elements $\pi_{M} \in Z(B)$ and $\pi_{M^{*}} \in Z(C)$ are invertible. Every indecomposable direct summand of $L_{0}$ has a proper subgroup of $\Delta D$ as a vertex and a trivial source; so does every indecomposable direct summand of $L_{0}^{*}$. We see from [6, Proposition 2.12 (iv)] that

$$
\pi_{M}=\pi_{L}+\pi_{L_{0}}, \quad \pi_{M^{*}}=\pi_{L^{*}}+\pi_{L_{0}^{*}} .
$$

Since the elements $\pi_{L_{0}}$ and $\pi_{L_{0}^{*}}$ are nilpotent by Lemma 2.7 , we have

$$
\pi_{L} \equiv \pi_{M} \quad(\bmod J(Z(B))), \quad \pi_{L^{*}} \equiv \pi_{M^{*}} \quad(\bmod J(Z(C)))
$$

so that the elements $\pi_{L} \in Z(B)$ and $\pi_{L^{*}} \in Z(C)$ are invertible.

The last assertion follows from Theorem 5.6.

Proposition 2.8 (i) The $(B, B)$-bimodule $B$ is isomorphic to a direct summand of $L \otimes_{C} L^{*}$.

(ii) As a $k\left[H \times H^{\mathrm{op}}\right]$-module

$$
L^{*} \otimes_{B} L \equiv C \oplus O\left(\mathscr{Y}\left(G \times H^{\mathrm{op}}, \Delta D, H \times H^{\mathrm{op}}\right)\right) .
$$

Proof. Theorems 1.1 and 5.3 imply that the block ideal $B$ is a direct summand of $L \otimes_{C} L^{*}$ as a $(B, B)$-bimodule; the block ideal $C$ is a direct summand of $L^{*} \otimes_{B} L$ as a $(C, C)$-bimodule. The assertion (ii) follows from the fact that

$$
\left.L^{*} \otimes_{B} L\right|_{H \times H^{\mathrm{op}}} L \equiv C \oplus O\left(\mathscr{Y}\left(G \times H^{\mathrm{op}}, \Delta D, H \times H^{\mathrm{op}}\right)\right) .
$$

Our $(B, C)$-bimodule $L(B, C)$ links source modules of the blocks $B$ and $C$ as follows.

Theorem 2.9 Assume that a source module $X$ of the block $B$ and a source module $Y$ of the block $C$ are in the Green correspondence with respect to $\left(G \times D^{\mathrm{op}}, \Delta D, H \times D^{\mathrm{op}}\right)$. Then the following hold.

(i) $L^{*} \otimes_{B} X \equiv Y \oplus O\left(\mathscr{Y}\left(G \times D^{\mathrm{op}}, \Delta D, H \times D^{\mathrm{op}}\right)\right)$.

(ii) $L \otimes_{C} Y \equiv X \oplus O\left(\mathscr{X}\left(G \times D^{\mathrm{op}}, \Delta D, H \times D^{\mathrm{op}}\right)\right)$.

(iii) If $D \triangleleft H$, then $L \otimes_{C} Y \simeq X$.

Proof. Let $\mathscr{X}=\mathscr{X}\left(G \times D^{\mathrm{op}}, \Delta D, H \times D^{\mathrm{op}}\right)$ and $\mathscr{Y}=\mathscr{Y}\left(G \times D^{\mathrm{op}}, \Delta D\right.$, $\left.H \times D^{\text {op }}\right)$. Notice that the source module $X$ is isomorphic to a direct summand of the tensor product $L \otimes_{C} L^{*} \otimes_{B} X$ and the source module $Y$ is isomorphic to a direct summand of the tensor product $L^{*} \otimes_{B} L \otimes_{C} Y$ because of Theorems 1.1 and 5.3. 
(i) The tensor product $L^{*} \otimes_{B} X$ is a direct summand of the restriction $H \times D^{\text {op }} X \equiv Y \oplus O(\mathscr{Y})$. If $L^{*} \otimes_{B} X$ is relatively $\mathscr{Y}$-projective, then $L \otimes_{C} L^{*} \otimes_{B} X$ is, as a direct summand of $k G \otimes_{k H} L^{*} \otimes_{B} X$, relatively $\mathscr{Y}$-projective; hence so is $X \mid L \otimes_{C} L^{*} \otimes_{B} X$, a contradiction. Therefore $L^{*} \otimes_{B} X$ is not relatively $\mathscr{Y}$-projective so that the assertion holds.

(ii) The tensor product $L \otimes_{C} Y$ is a direct summand of the induced module $k\left[G \times D^{\mathrm{op}}\right] \otimes_{C} Y \equiv X \oplus O(\mathscr{X})$. If $L \otimes_{C} Y$ is relatively $\mathscr{X}$-projective, then $L^{*} \otimes_{B} L \otimes_{C} Y$ is, as a direct summand of the restriction $H \times D^{\text {op }} L \otimes_{C} Y$, relatively $\mathscr{Y}$-projective; hence so is $Y \mid L^{*} \otimes_{B} L \otimes_{C} Y$, a contradiction. Therefore $L \otimes_{C} Y$ is not relatively $\mathscr{X}$-projective so that the assertion holds.

(iii) From the fact (ii) we can write

$$
L \otimes_{C} Y \simeq X \oplus X_{0}, \quad X_{0} \equiv O(\mathscr{X}) .
$$

Since $L$ has $\Delta D$ as a vertex and a trivial source, there exists an indecomposable direct summand $W$ of the induced module $k\left[G \times D^{\mathrm{op}}\right] \otimes_{k \Delta D} k$ with vertex $\Delta D$ such that $L$ is a direct summand of the induced module $k[G \times$ $\left.H^{\mathrm{op}}\right] \otimes_{k\left[G \times D^{\mathrm{op}}\right]} W$. Then we obtain

$$
\begin{aligned}
& X \oplus X_{0} \simeq L \otimes_{k H} Y \mid k\left[G \times H^{\mathrm{op}}\right] \otimes_{k\left[G \times D^{\mathrm{op}}\right]} W \otimes_{k H} Y \\
& \mid W \otimes_{k D} k H_{k D}=\bigoplus_{t \in D \backslash H}^{(1, t)} W
\end{aligned}
$$

This implies that $X_{0}=0$ because the module ${ }^{(1, t)} W$ has a vertex conjugate to $\Delta D$.

The $(B, C)$-bimodule $L$ is splendid with respect to source modules of $B$ and $C$ which are in Green correspondence. Namely

Theorem 2.10 Let $X$ and $Y$ be as in Theorem 2.9. Then the (B, $C)$-bimodule $L(B, C)$ is isomorphic with a direct summand of the tensor product $X \otimes_{k D} Y^{*}$.

Proof. We argue with the same notation as in the proof of Theorem 2.9. Tensoring $Y^{*}$ to the both sides of $L \otimes_{C} Y \simeq X \oplus X_{0}$ on the right, we obtain

$$
L \mid L \otimes_{C} Y \otimes_{k D} Y^{*} \simeq X \otimes_{k D} Y^{*} \oplus X_{0} \otimes_{k D} Y^{*}
$$

since $C \mid Y \otimes_{k D} Y^{*}$. Note that $X_{0} \otimes_{k D} Y^{*}$ is a direct summand of $X_{0} \otimes_{k D} k H=$ $k\left[G \times H^{\text {op }}\right] \otimes_{k\left[G \times D^{\text {op }}\right]} X_{0}$. If $L \mid X_{0} \otimes_{k D} Y^{*}$, then $L$ is also projective relative to $\mathscr{X}$. However, this contradicts to the fact the module $L$ has $\Delta D$ as a vertex. Therefore we conclude that $L \mid X \otimes_{k D} Y^{*}$.

Relatively projective elements associated with the tensor products of the bimodules $L, X$ and $Y$ and their duals are all invertible if $X$ and $Y$ are in Green correspondence. 
Theorem 2.11 Let $X$ and $Y$ be as in Theorem 2.9.

(i) Relatively projective elements $\pi_{L \otimes_{C} Y} \in Z(B)$ and $\pi_{Y^{*} \otimes_{C} L^{*}} \in Z(k D)$ are invertible.

(ii) Relatively projective elements $\pi_{X^{*} \otimes_{B} L \otimes_{C} Y} \in Z(k D)$ and $\pi_{X^{*} \otimes_{B} L} \in Z(k D)$ are invertible.

(iii) Relatively projective elements $\pi_{Y^{*} \otimes_{C} L^{*} \otimes_{B} X} \in Z(k D)$ and $\pi_{L^{*} \otimes_{B} X} \in Z(C)$ are invertible.

Proof. Again we argue with the same notation as in the proof of Theorem 2.9 .

(i) We see from Lemma 2.7 that the relatively projective elements $\pi_{X_{0}} \in$ $Z(B)$ and $\pi_{X_{0}^{*}} \in Z(k D)$ are nilpotent. Therefore, a similar argument in the proof of Theorem 1.1 shows that $\mathrm{t} \pi_{X \oplus X_{0}}$ and $\pi_{X^{*} \oplus X_{0}^{*}}$ are invertible; hence so are the elements $\pi_{L \otimes_{C} Y}$ and $\pi_{Y^{*} \otimes_{C} L^{*}}$.

(ii) Let $t_{X^{*}}: H H^{*}(B) \rightarrow H H^{*}(k D)$ be the transfer map associated with the $(k D, B)$-bimodule $X^{*}$. Then, since $\pi_{X^{*} \otimes_{B} L \otimes_{C} Y}=t_{X^{*}}\left(\pi_{L \otimes_{C} Y}\right)$ and the element $\pi_{L \otimes_{C} Y}$ is invertible, Kawai and Sasaki [4, Proposition 3.5] implies that $\pi_{X^{*} \otimes_{B} L \otimes_{C} Y}$ is invertible. We also have that $\pi_{X^{*} \otimes_{B} L}=t_{X^{*}}\left(\pi_{L}\right)$ and the element $\pi_{L}$ is invertible; hence again [4, Proposition 3.5] implies that the element $\pi_{X^{*} \otimes_{B} L}$ is invertible.

(iii) In the direct sum decomposition $Y^{*} \otimes_{C} L^{*} \otimes_{B} X \simeq X^{*} \otimes_{B} X \oplus X_{0}^{*} \otimes_{B} X$, every indecomposable direct summand of $X_{0}^{*} \otimes_{B} X$ has a proper subgroup of $\Delta D$ as a vertex and a trivial source. Therefore the element $\pi_{X_{0}^{*} \otimes_{B} X} \in Z(k D)$ is nilpotent; hence we see that $\pi_{Y^{*} \otimes_{C} L^{*} \otimes_{B} X} \equiv \pi_{X^{*} \otimes_{B} X}(\bmod J(Z(k D)))$ and that the element $\pi_{Y^{*} \otimes_{C} L^{*} \otimes_{B} X}$ is invertible. Furthermore, since $\pi_{Y^{*} \otimes_{C} L^{*} \otimes_{B} X}=$ $t_{Y^{*}}\left(\pi_{L^{*} \otimes_{B} X}\right)$, where $t_{Y^{*}}: H H^{*}(k D) \rightarrow H H^{*}(C)$ is the transfer map associated with $Y^{*}$, again [4, Proposition 3.5] implies that the element $\pi_{L^{*} \otimes_{B} X}$ is invertible.

\section{Cohomology Algebras of Block Ideals}

We continue our argument under Situation (BC); let $L=L(B, C)$. We assume that a source module $X$ of $B$ and a source module $Y$ of $C$ are in the Green correspondence with respect to $\left(G \times D^{\mathrm{op}}, \Delta D, H \times D^{\mathrm{op}}\right)$. Let us take a Sylow $C$-subpair $\left(D, b_{D}\right)$ satisfying $b_{D} Y(D) \neq 0$. Then Proposition 2.3 says that $\left(D, b_{D}\right)$ is also a Sylow $B$-subpair and that $b_{D} X(D) \neq 0$. The cohomology algebras of blocks $B$ and $C$ with respect to the Sylow subpair $\left(D, b_{D}\right)$ satisfy

$$
H^{*}(G, B ; X) \stackrel{\delta_{D}}{\mapsto} H H_{X^{*}}^{*}(k D), \quad H^{*}(H, C ; Y) \stackrel{\delta_{D}}{\mapsto} H H_{Y^{*}}^{*}(k D) .
$$


We know from Theorems 1.1 and 2.11 that the relatively projective elements are invertible; hence we obtain from Kawai and Sasaki [4, Proposition 2.2] the following commutative diagram:

$$
\begin{aligned}
& H H_{Y^{*} \otimes_{C} L^{*}}^{*}(k D) \underset{\overbrace{R_{Y^{*}}}}{\stackrel{R_{Y}}{\sharp}} H_{\langle} H H_{L^{*}}^{*}(C) \cap H H_{Y}^{*}(C)
\end{aligned}
$$

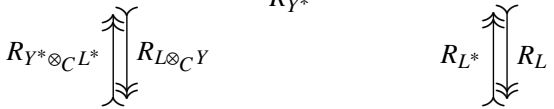

$$
\begin{aligned}
& H H_{L \otimes_{C} Y}^{*}(B)=H H_{L \otimes_{C} Y}^{*}(B)
\end{aligned}
$$

The diagram above together with Theorem 2.11, [4, Lemma 4.4] and Linckelmann [6, Corollary 3.8] yields the following.

Lemma 3.1 The following diagram commutes:

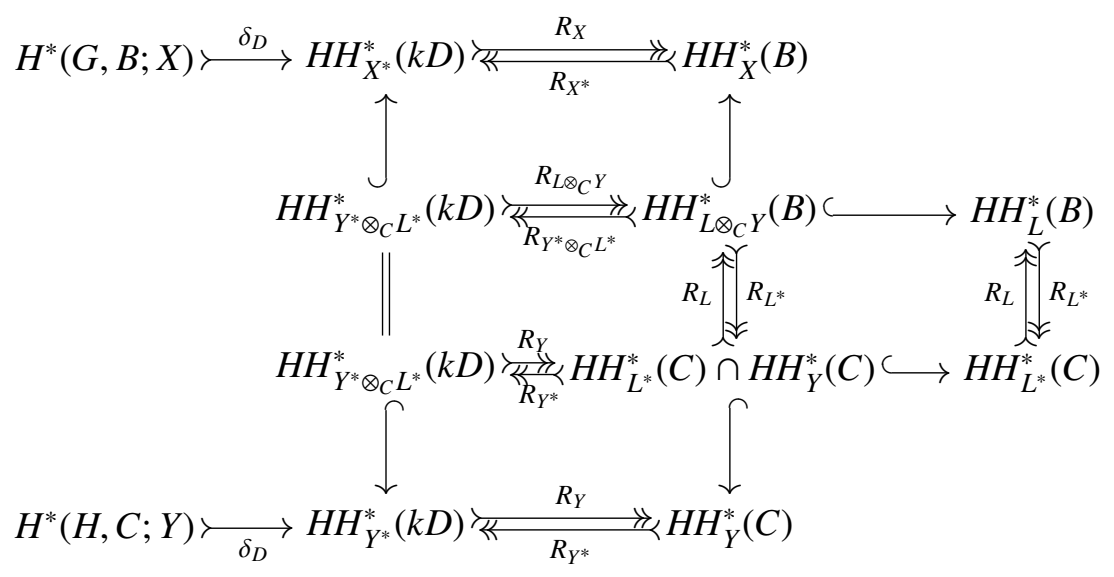

In particular

Corollary 3.2 If $D$ is normal in $H$, then we have the following commutative diagram

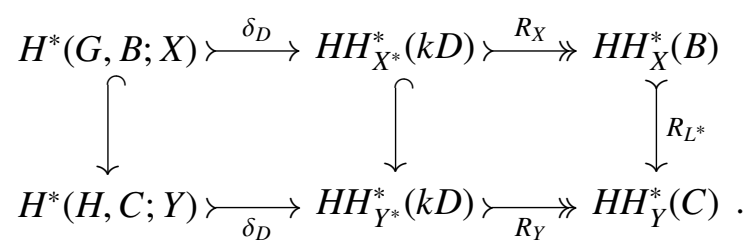

Namely, the inclusion map $H^{*}(G, B ; X) \hookrightarrow H^{*}(H, C ; Y)$ is the restriction of the normalized transfer map $R_{L^{*}}$.

Proof. Theorem 2.9 and [5, Proposition 2.3] and Lemma 3.1 gives rise to the diagram. 
We generalize this to the case that $D$ is not necessarily normal in $H$. In Kawai and Sasaki [4] we discussed under the following situation:

Let $b \in Z(k G)$ and $c \in Z(k H)$ be block idempotents and assume that the Brauer correspondent $c^{G}$ is defined and $c^{G}=b$ and moreover that the blocks $b$ and $c$ have a common defect group $D$. Let $X=c k G b$. Let $Y=k G i$ be a source module of $b$ and let $Z=k H j$ be a source module of $c$ such that

$$
Y=k G i \mid X^{*} \otimes_{k G b} Z=b k G j .
$$

We considered bimodules

$$
\begin{aligned}
c k G i & =X \otimes_{k G b} Y, & i k G c & =Y^{*} \otimes_{k G b} X^{*}, \\
b k G j & =X^{*} \otimes_{k H c} Z, & j k G b & =Z^{*} \otimes_{k H c} X, \\
i k G j & =Y^{*} \otimes_{k G b} X^{*} \otimes_{k H c} Z, & j k G i & =Z^{*} \otimes_{k H c} X \otimes_{k G b} Y .
\end{aligned}
$$

We showed in [4, Theorem 1.1] that the relatively projective elements associated with the bimodules above are all invertible.

In this paper we take $L^{*}$ instead of $X=c k G b$ above; we have gotten the corresponding facts to the above in Theorem 2.11; hence the conclusions in [4, Section 4] and [4, Theorem 1.2] hold by replacing $c k G b$ there with our $(C, B)$-bimodule $L^{*}$.

Thus the argument in [4, Proof of Theorem 1.2] yields

Lemma 3.3 We have the following commutative diagram:

$$
\begin{aligned}
& H H_{X^{*} \otimes_{B} L \otimes_{C} Y}^{*}(k D) \underset{R_{X^{*}}}{\stackrel{R_{X}}{\rightleftarrows}} H_{L} H H_{L \otimes_{C} Y}^{*}(B)
\end{aligned}
$$

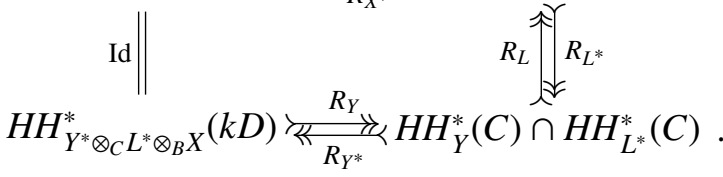

We obtain from Lemmas 3.1 and 3.3 that

$$
\begin{aligned}
H H_{Y^{*} \otimes_{C} L^{*} \otimes_{B} X}^{*}(k D) & =H H_{X^{*} \otimes_{B} L \otimes_{C} Y}^{*}(k D) \\
& =R_{X^{*}}\left(H H_{L \otimes_{C} Y}^{*}(B)\right)=H H_{Y^{*} \otimes_{C} L^{*}}^{*}(k D) .
\end{aligned}
$$

Consequently we have

Proposition 3.4 Under Situation (BC) assume that a source module $X$ of $B$ and a source module $Y$ of $C$ are in the Green correspondence with respect to 
$\left(G \times D^{\mathrm{op}}, \Delta D, H \times D^{\mathrm{op}}\right)$. Then we have the following commutative diagram

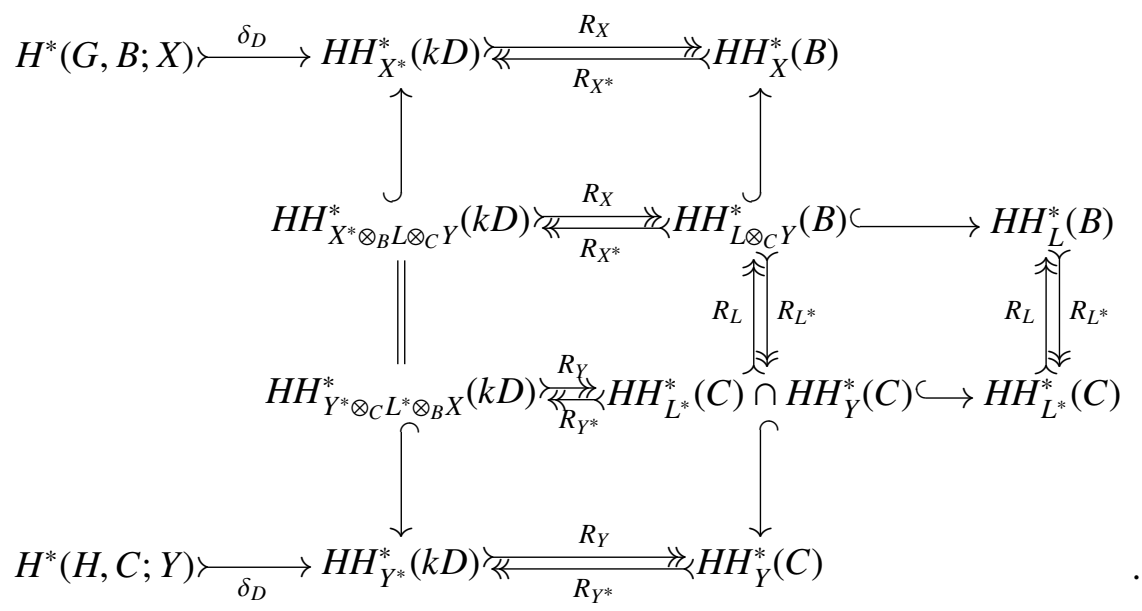

We are now in a position to prove Theorem 1.2.

Proof of Theorem 1.2. We know from Theorem 2.10 that $L \mid X \otimes_{k D} Y^{*}=$ $k G i \otimes_{k D} j k H$. For a subgroup $R \leqslant D$ we let $\left(R, b_{R}\right) \leqslant\left(D, b_{D}\right)$ be the $B$-subpair and let $\left(R, c_{R}\right) \leqslant\left(D, b_{D}\right)$ be the $C$-subpair. Then Kessar, Linckelmann and Robinson [5, Proposition 2.3] implies for $R \leqslant D$ that

$$
E_{H}\left(\left(R, c_{R}\right),\left(D, b_{D}\right)\right) \subseteq E_{G}\left(\left(R, b_{R}\right),\left(D, b_{D}\right)\right) .
$$

Therefore we have from Linckelmann [6, Theorem 5.7] that $H^{*}(G, B ; X) \subseteq$ $H^{*}(H, C ; Y)$ and that $\delta_{D} H^{*}(G, B ; X) \subseteq H H_{X^{*} \otimes_{B} L \otimes_{C} Y}(k D)$. Then [4, Proof of Theorem 1.2] gives the left rectangle of the diagram; we get from the diagram just before the theorem the right rectangle of the diagram.

\section{Green Correspondence and Block Varieties of Modules}

In this section we investigate relationship between the Green correspondence of indecomposable modules and the Brauer correspondence of blocks.

We argue under Situation (BC); let $L=L(B, C)$. We let moreover $P \leqslant D$ and assume that the subgroup $H$ contains $N_{G}(P)$.

Proposition 4.1 Assume that an indecomposable B-module $U$ and an indecomposable $C$-module $V$ have vertices in $\mathscr{A}(G, P, H)$ and are in the Green correspondence with respect to $(G, P, H)$. Then the followings hold:

$$
L \otimes_{C} V \equiv U \oplus O(\mathscr{X}(G, P, H)), \quad L^{*} \otimes_{B} U \equiv V \oplus O(\mathscr{Y}(G, P, H)) .
$$


Proof. Since the module $V$ lies in the block $C$, we have

$$
L \otimes_{C} V \mid k G \otimes_{k H} V \equiv U \oplus O(\mathscr{X}(G, P, H)) .
$$

If $L \otimes_{C} V$ is relatively $\mathscr{X}(G, P, H)$-projective, then we would have

$$
V\left|L^{*} \otimes_{B} L \otimes_{C} V\right|{ }_{H} L \otimes_{C} V \equiv O(\mathscr{Y}(G, P, H)),
$$

a contradiction. Thus $L \otimes_{C} V$ is not relatively $\mathscr{X}(G, P, H)$-projective; hence we have that $L \otimes_{C} V \equiv U \oplus O(\mathscr{X}(G, P, H))$. Let us write

$$
L \otimes_{C} V \simeq U \oplus U_{0}, \quad U_{0} \equiv O(\mathscr{X}(G, P, H)) .
$$

Tensor with $L^{*} \otimes_{B}-$ to obtain

$$
V \mid L^{*} \otimes_{B} L \otimes_{C} V \simeq L^{*} \otimes_{B} U \oplus L^{*} \otimes_{B} U_{0} .
$$

Because $L^{*} \otimes_{B} U_{0} \mid{ }_{H} U_{0} \equiv O(\mathscr{Y}(G, P, H))$, we see that $V \mid L^{*} \otimes_{B} U$. This together with the fact that $L^{*} \otimes_{B} U \mid{ }_{H} U \equiv V \oplus O(\mathscr{Y}(G, P, H))$ implies that $L^{*} \otimes_{B} U \equiv V \oplus O(\mathscr{Y}(G, P, H))$, as required.

Here we recall a general fact. For a family $\mathscr{F}$ of subgroups of a finite group $G$ and a subgroup $H$ of $G$ we set

$$
H \wedge_{G} \mathscr{F}=\left\{H \cap{ }^{g} F \mid g \in G, F \in \mathscr{F}\right\} .
$$

Mackey double coset formula yields

Lemma 4.2 Let $\mathscr{F}$ be a family of subgroups of a finite group $G$. If a $k G$ module $U$ is relatively $\mathscr{F}$-projective, then for a subgroup $H$ of $G$ the $\mathrm{kH}$ module ${ }_{H} U$ is relatively $H \wedge_{G} \mathscr{F}$-projective.

Lemma 4.3 (i) Assume that a direct summand $W$ of the $k\left[H \times D^{\mathrm{op}}\right]$-module $k H$ is relatively $\mathscr{Y}\left(G \times D^{\mathrm{op}}, \Delta D, H \times D^{\mathrm{op}}\right)$-projective. Then for a subgroup $Q \in \mathscr{A}(G, P, H)$ and a $k Q$-module $S$, the $k Q$-module ${ }_{Q} W \otimes_{k Q} S$ is relatively $\left\{Q \cap{ }^{g} Q \mid g \in G \backslash H\right\}$-projective.

(ii) Assume that a direct summand $Z$ of the $k\left[G \times D^{\mathrm{op}}\right]$-module $k G$ is relatively $\mathscr{X}\left(G \times D^{\mathrm{op}}, \Delta D, H \times D^{\mathrm{op}}\right)$-projective. Then for a subgroup $Q \in$ $\mathscr{A}(G, P, H)$ and a $k Q$-module $S$, the $k Q$-module ${ }_{Q} Z \otimes_{k Q} S$ is relatively $\left\{Q \cap{ }^{g} Q \mid g \in G \backslash H\right\}$-projective.

Proof. Let $\mathscr{T}=\left\{T \leqslant Q \times Q^{\mathrm{op}} \cap{ }^{(s, a)} \Delta D \mid(s, a) \in G \times D^{\mathrm{op}} \backslash H \times D^{\mathrm{op}}\right\}$.

(i) Let $\mathscr{Y}=\mathscr{Y}\left(G \times D^{\mathrm{op}}, \Delta D, H \times D^{\mathrm{op}}\right)$. The $k\left[Q \times Q^{\mathrm{op}}\right]$-module $Q \times Q^{\mathrm{op}} W$ is relatively $Q \times Q^{\text {op }} \wedge_{H \times D^{\text {op }}} \mathscr{Y}$-projective. We show that

$$
Q \times Q^{\text {op }} \wedge_{H \times D^{\text {op }}} \mathscr{Y} \subseteq \mathscr{T} .
$$


Take a subgroup $R \in \mathscr{Y}$. There exists an element $\left(s^{\prime}, a^{\prime}\right) \in G \times D^{\text {op }} \backslash H \times D^{\text {op }}$ for which $R \leqslant H \times D^{\mathrm{op}} \cap^{\left(s^{\prime}, a^{\prime}\right)} \Delta D$. Then we have for an element $(t, b) \in H \times D^{\text {op }}$ that

$$
Q \times Q^{\mathrm{op}} \cap^{(t, b)} R \leqslant Q \times Q^{\mathrm{op}} \cap^{(t, b)\left(s^{\prime}, a^{\prime}\right)} \Delta D .
$$

Since $(t, b)\left(s^{\prime}, a^{\prime}\right) \in G \times D^{\text {op }} \backslash H \times D^{\text {op }}$, we obtain for some element $(s, a) \in$ $G \times D^{\text {op }} \backslash H \times D^{\text {op }}$ that

$$
Q \times Q^{\mathrm{op}} \cap^{(t, b)} R \leqslant Q \times Q^{\mathrm{op}} \cap^{(s, a)} \Delta D .
$$

Thus we see that $Q \times Q^{\text {op }} \wedge_{H \times D^{\text {op }}} \mathscr{Y} \subseteq \mathscr{T}$.

Notice for an element $(s, a) \in G \times D^{\mathrm{op}} \backslash H \times D^{\mathrm{op}}$ that

$$
Q \times Q^{\mathrm{op}} \cap{ }^{(s, a)} \Delta D={ }^{(s, a)} \Delta\left(Q^{s} \cap{ }^{a} Q\right) .
$$

Now an indecomposable direct summand $W^{\prime}$ of the $k\left[Q \times Q^{\text {op }}\right]$-module $Q \times Q^{\text {op } W}$ is isomorphic for some element $x \in H$ to $k[Q x Q]$, which has ${ }^{(1, x)} \Delta\left(Q \cap{ }^{x} Q\right)$ as a vertex. Because $W^{\prime}$ is relatively $\mathscr{T}$-projective, there exists an element $(s, a) \in G \times D^{\mathrm{op}} \backslash H \times D^{\mathrm{op}}$ for which

$$
{ }^{(1, x)} \Delta\left(Q \cap{ }^{x} Q\right) \leqslant Q \times Q^{\text {op }} Q \times Q^{\text {op }} \cap{ }^{(s, a)} \Delta D={ }^{(s, a)} \Delta\left(Q^{s} \cap{ }^{a} Q\right) .
$$

Hence we have that $Q \cap{ }^{x} Q \leqslant Q \quad Q \cap{ }^{s a} Q$. Since $s a \in G \backslash H$, we have that

$$
Q \cap{ }^{x} Q \leqslant Q Q \cap{ }^{g} Q
$$

for some element $g \in G \backslash H$.

Now, because $W^{\prime} \otimes_{k Q} S \simeq k[Q x Q] \otimes_{k Q} S \simeq k Q \otimes_{k[Q \cap Q]} x \otimes S$, the $k Q-$ module $W^{\prime} \otimes_{k} S$ is relatively $Q \cap ' Q$-projective. Thus we conclude that $W^{\prime} \otimes_{k} Q$ $S$ is relatively $Q \cap{ }^{g} Q$-projective for some element $g \in G \backslash H$.

(ii) We set $\mathscr{X}=\mathscr{X}\left(G \times D^{\mathrm{op}}, \Delta D, H \times D^{\mathrm{op}}\right)$. The $k\left[Q \times Q^{\mathrm{op}}\right]$-module $Q \times Q^{\text {op }} Z$ is relatively $Q \times Q^{\text {op }} \wedge_{G \times D^{\text {op }}} \mathscr{X}$-projective. Take a subgroup $R \in \mathscr{X}$. Then there exists an element $\left(s^{\prime}, a^{\prime}\right) \in G \times D^{\text {op }} \backslash H \times D^{\text {op }}$ for which $R \leqslant$ $\Delta D \cap{ }^{\left(s^{\prime}, a^{\prime}\right)} \Delta D$. For an element $(t, b) \in H \times D^{\text {op }}$, we have

$$
Q \times Q^{\mathrm{op}} \cap^{(t, b)} R \leqslant Q \times Q^{\mathrm{op}} \cap{ }^{(t, b)} \Delta D \cap{ }^{(t, b)\left(s^{\prime}, a^{\prime}\right)} \Delta D .
$$

Either $(t, b)$ or $(t, b)\left(s^{\prime}, a^{\prime}\right)$ does not belong to $H \times D^{\text {op }}$ so that we have for some element $(s, a) \in G \times D^{\text {op }} \backslash H \times D^{\text {op }}$ that

$$
Q \times Q^{\mathrm{op}} \cap{ }^{(t, b)} R \leqslant Q \times Q^{\mathrm{op}} \cap{ }^{(s, a)} \Delta D .
$$

Namely we see that $Q \times Q^{\text {op }} \wedge_{G \times D^{\text {op }}} \mathscr{X} \subseteq \mathscr{T}$.

Now an indecomposable direct summand $Z^{\prime}$ of $Q \times Q^{\text {op }} Z$ is isomorphic for some element $x \in G$ to $k[Q x Q]$, which has ${ }^{(1, x)} \Delta\left(Q \cap{ }^{x} Q\right)$ as a vertex. The module $Z^{\prime}$ is relatively $\mathscr{T}$-projective; hence we have that

$$
{ }^{(1, x)} \Delta\left(Q \cap{ }^{x} Q\right) \leqslant Q \times Q^{\text {op }} Q \times Q^{\text {op }} \cap{ }^{(s, a)} \Delta D={ }^{(s, a)} \Delta\left(Q^{s} \cap{ }^{a} Q\right)
$$


for some element $(s, a) \in G \times D^{\mathrm{op}} \backslash H \times D^{\mathrm{op}}$. Therefore, it follows that $Q \cap{ }^{x} Q \leqslant Q Q{ }^{s a} Q$. Since $s a \in G \backslash H$, we see that

$$
Q \cap{ }^{x} Q \leqslant Q Q \cap{ }^{g} Q
$$

for some element $g \in G \backslash H$.

Now, because $Z^{\prime} \otimes_{k Q} S \simeq k[Q x Q] \otimes_{k Q} S=k Q \otimes_{k[Q \cap Q]} x \otimes S$, the tensor product $Z^{\prime} \otimes_{k Q} S$ is relatively $Q \cap ' Q$-projective. Consequently the tensor product $Z^{\prime} \otimes_{k} S$ is relatively $Q \cap{ }^{g} Q$-projective for some element $g \in G \backslash H$.

Proposition 4.4 We let a source module $X$ of the block ideal $B$ and a source module $Y$ of the block ideal $C$ be in the Green correspondence with respect to $\left(G \times D^{\mathrm{op}}, \Delta D, H \times D^{\mathrm{op}}\right)$. Assume that an indecomposable $k G$-module $U$ lying in $B$ and an indecomposable $k H$-module $V$ lying in $C$ have vertices in $\mathscr{A}(G, P, H)$ and are in the Green correspondence with respect to $(G, P, H)$. Then the following hold.

(i) If $Q \in \mathscr{A}(G, P, H)$ is a $Y$-vertex of $V$ and $S$ is a $(Q, Y)$-source of $V$, then $Q$ is an $X$-vertex of $U$ and $S$ is a $(Q, X)$-source of $U$.

(ii) If $Q \in \mathscr{A}(G, P, H)$ is an $X$-vertex of $U$ and $S$ is a $(Q, X)$-source of $U$, then $Q$ is a $Y$-vertex of $V$ and $S$ is a $(Q, Y)$-source of $V$.

Proof. We first note for any $g \in G \backslash H$ that $Q \cap{ }^{g} Q$ is a proper subgroup of $Q$.

Now we let

$$
\begin{aligned}
L^{*} \otimes_{B} X \simeq Y \oplus Y_{0}, & Y_{0} \equiv O\left(\mathscr{Y}\left(G \times D^{\mathrm{op}}, \Delta D, H \times D^{\mathrm{op}}\right)\right), \\
L \otimes_{C} Y \simeq X \oplus X_{0}, & X_{0} \equiv O\left(\mathscr{X}\left(G \times D^{\mathrm{op}}, \Delta D, H \times D^{\mathrm{op}}\right)\right), \\
L^{*} \otimes_{B} U \simeq V \oplus V_{0}, & V_{0} \equiv O(\mathscr{Y}(G, P, H)), \\
L \otimes_{C} V \simeq U \oplus U_{0}, & U_{0} \equiv O(\mathscr{X}(G, P, H)) .
\end{aligned}
$$

(i) Let us show that $S \mid{ }_{Q} X^{*} \otimes_{B} U$. Because

$$
\begin{aligned}
& X^{*} \otimes_{B} L \otimes_{C} V \simeq X^{*} \otimes_{B} U \oplus X^{*} \otimes_{B} U_{0}, \\
& X^{*} \otimes_{B} L \otimes_{C} V \simeq Y^{*} \otimes_{C} V \oplus Y_{0}^{*} \otimes_{C} V
\end{aligned}
$$

and $S \mid{ }_{Q} Y^{*} \otimes_{C} V$, the module $S$ is isomorphic with an indecomposable direct summand of either ${ }_{Q} X^{*} \otimes_{B} U$ or ${ }_{Q} X^{*} \otimes_{B} U_{0}$. The $k Q$-module ${ }_{Q} X^{*} \otimes_{B} U_{0}$ is a direct summand of the $k Q$-module $Q_{Q} U_{0}$, which is projective relative to the family $Q \wedge_{G} \mathscr{X}(G, P, H)$. We see for an element $g \in G$ and an element $t \in G \backslash H$ that $Q \cap{ }^{g}\left(P \cap{ }^{t} P\right) \leqslant Q \cap{ }^{g t} P \leqslant P \cap{ }^{g t} P$ so that the $k Q$-module ${ }_{Q} X^{*} \otimes_{B} U_{0}$ has no indecomposable direct summand having $Q$ as a vertex. Therefore we obtain that $S \mid{ }_{Q} X^{*} \otimes_{B} U$, as claimed. 
Next we show that $Q$ is an $X$-vertex of $U$. It follows that

$$
U\left|L \otimes_{C} V\right| L \otimes_{C} Y \otimes_{k Q} S \simeq X \otimes_{k Q} S \oplus X_{0} \otimes_{k Q} S .
$$

If $U \mid X_{0} \otimes_{k Q} S$, then we would have

$$
S\left|{ }_{Q} X^{*} \otimes_{B} U\right|{ }_{Q} U \mid{ }_{Q} X_{0} \otimes_{k Q} S .
$$

However, Lemma 4.3 (ii) says that ${ }_{Q} X_{0} \otimes_{k Q} S$ is projective relative to the family $\left\{Q \cap{ }^{g} Q \mid g \in G \backslash H\right\}$, a contradiction.

(ii) We show that $S \mid{ }_{k Q} Y^{*} \otimes_{C} V$. Because

$$
\begin{aligned}
& Y^{*} \otimes_{C} L^{*} \otimes_{B} U \simeq Y^{*} \otimes_{C} V \oplus Y^{*} \otimes_{C} V_{0}, \\
& Y^{*} \otimes_{C} L^{*} \otimes_{B} U \simeq X^{*} \otimes_{B} U \oplus X_{0}^{*} \otimes_{B} U
\end{aligned}
$$

and $S \mid{ }_{Q} X^{*} \otimes_{B} U$, the $k Q$-module $S$ is isomorphic to a direct summand of either $Y^{*} \otimes_{C} V$ or ${ }_{Q} Y^{*} \otimes_{C} V_{0}$. The $k Q$-module ${ }_{Q} Y^{*} \otimes_{C} V_{0}$ is projective relative to the family $Q \wedge_{H} \mathscr{Y}(G, P, H)$. We see for an element $s \in H$ and an element $t \in G \backslash H$ that $Q \cap{ }^{s}\left(H \cap{ }^{t} P\right) \leqslant Q \cap{ }^{s t} P \leqslant P \cap{ }^{s t} P$ so that the $k Q$-module ${ }_{Q} Y^{*} \otimes_{C} V_{0}$ has no indecomposable direct summand having $Q$ as a vertex. Therefore we see that $S \mid Q_{Q} Y^{*} \otimes_{C} V$, as desired.

Next we prove that $Q$ is a $Y$-vertex of $V$. Because

$$
V\left|L^{*} \otimes_{B} U\right| L^{*} \otimes_{B} X \otimes_{k Q} S \simeq Y \otimes_{k Q} S \oplus Y_{0} \otimes_{k Q} S
$$

the $k H$-module $V$ is a direct summand of either $Y \otimes_{k Q} S$ or $Y_{0} \otimes_{k Q} S$. If $V \mid Y_{0} \otimes_{k Q} S$, then we would have

$$
\left.S\left|{ }_{Q} Y^{*} \otimes_{C} V\right|_{Q} V\right|_{Q} Y_{0} \otimes_{k Q} S .
$$

However, Lemma 4.3 (i) says that ${ }_{Q} Y_{0} \otimes_{k} S$ is projective relative to the family $\left\{Q \cap{ }^{g} Q \mid g \in G \backslash H\right\}$, a contradiction.

Since $\left.L\right|_{G \times H^{\text {op }} B}$ and $L$ has $\Delta D$ as a vertex, an indecomposable direct summand of $G \times D^{\mathrm{op}} L$ has $\Delta D$ as a vertex, which is a source module of the block $B$.

Proposition 4.5 We take a source module $X$ of the block ideal $B$ as a direct summand of the $k\left[G \times D^{\mathrm{op}}\right]$-module $G \times D^{\mathrm{op}} L$. Assume that an indecomposable $B$-module $U$ has an $X$-vertex belonging to $\mathscr{A}(G, P, H)$. Then the Green correspondent $V$ of $U$ to $H$ with respect to $(G, P, H)$ lies in the block $C$.

Proof. Let $Q \in \mathscr{A}(G, P, H)$ be an $X$-vertex of $U$ and $S$ a $(Q, X)$-source of $U$. Our choice of the source module $X$ implies that

$$
S\left|{ }_{Q} X^{*} \otimes_{B} U\right|{ }_{Q} L^{*} \otimes_{B} U
$$


Therefore an indecomposable direct summand $V^{\prime}$ of $L^{*} \otimes_{B} U$ has $Q$ as vertex, which must be isomorphic with $V$ by Green correspondence:

$$
V \simeq V^{\prime} \mid L^{*} \otimes_{B} U
$$

and that the Green correspondent $V$ lies in the block $C$.

Proof of Theorem 1.3. (i) Let $Q \in \mathscr{A}(G, P, H)$ be an $X$-vertex of $U$. Then Proposition 4.5 says that the Green correspondent $V$ lies in the block $C$. Proposition 4.4 says that $Q$ is a $Y$-vertex of $V$ and we can take a common $(Q, X)$-source of $U$ and $(Q, Y)$-source of $V$, which we denote by $S$. We write the restrictions of $\operatorname{res}_{Q}^{D}: H^{*}(D, k) \rightarrow H^{*}(Q, k)$ to $H^{*}(G, B ; X)$ and $H^{*}(H, C ; Y)$ as follows:

$$
r_{B, Q}: H^{*}(G, B ; X) \rightarrow H^{*}(Q, k), \quad r_{C, Q}: H^{*}(H, C ; Y) \rightarrow H^{*}(Q, k) .
$$

Then Benson and Linckelmann [2, Theorem 1.1] implies that

$$
V_{G, B}(U)=r_{B, Q}{ }^{*}\left(V_{Q}(S)\right), \quad V_{H, C}(V)=r_{C, Q}{ }^{*}\left(V_{Q}(S)\right) .
$$

Since $r_{B, Q^{*}}=\iota^{*} \circ r_{C, Q^{*}}$, we obtain that

$$
V_{G, B}(U)=\iota^{*} V_{H, C}(V) .
$$

(ii) It is well known that the Green correspondent $U$ of $V$ lies in the block $B=C^{G}$ without any assumption on $Y$-vertices. Let $Q \in \mathscr{A}(G, P, H)$ be a $Y$ vertex of $V$. Then Proposition 4.4 says that $Q$ is an $X$-vertex of $U$ and we can take a common $(Q, X)$-source of $U$ and $(Q, Y)$-source of $V$. Thus the same argument as in the above applies.

Example. (cf Benson and Linckelmann [2, Corollary 1.4]) Let $B$ be a block ideal of $k G$ and $D \leqslant G$ a defect group of $B$. Let $X=k G i$ be a source module of $B$. Let $U$ be an indecomposable $B$-module and $Q$ an $X$-vertex of $U$ and $S$ a $(Q, X)$-source of $U$.

Assume that $Q$ is normal in $D$. Let $H=N_{G}(Q)$. Let $P \leqslant D$ and assume that $H$ contains $N_{G}(P)$ and that $Q \in \mathscr{A}(G, P, H)$.

Let $\left(D, b_{D}\right)$ be a Sylow $B$-subpair such that $b_{D} X(D)=X(D)$ and let $\left(Q, b_{Q}\right) \leqslant\left(D, b_{D}\right)$. Let $C$ be a unique block ideal of $k H$ covering the block ideal $b_{Q}$ of $k Q C_{G}(Q)$.

Because $Q$ is normal in $D$, we see that $H=N_{G}(Q) \geqslant D C_{G}(D)$. We also know that $C^{G}=B$ and $D$ is a defect group of the block $C$ and $\left(D, b_{D}\right)$ is a Sylow $C$-subpair.

We let $K=D C_{G}(D)$. The $k\left[K \times D^{\mathrm{op}}\right]$-module $Z=k K \mathrm{Br}_{D}(i)$ is a source module of $b_{D}$ and the source module $X$ of $B$ is the Green correspondent of $Z$ with respect to $\left(G \times D^{\mathrm{op}}, \Delta D, K \times D^{\mathrm{op}}\right)$. Let $Y$ be the Green correspondent of 
$Z$ with respect to $\left(H \times D^{\mathrm{op}}, \Delta D, K \times D^{\mathrm{op}}\right) ; Y$ is a source module of the block $C=b_{D}{ }^{H}$ by Proposition 2.3; $X$ and $Y$ are in the Green correspondence with respect to $\left(G \times D^{\mathrm{op}}, \Delta D, H \times D^{\mathrm{op}}\right)$.

Theorem 1.2 (ii) implies that $H^{*}(G, B ; X) \subseteq H^{*}(H, C ; Y)$.

Let $V$ be the Green correspondent of $U$ with respect to $(G, P, H)$. Then we have from Theorem 1.3 that

$$
V_{G, B}(U)=\iota^{*} V_{H, C}(V)
$$

\section{Appendix-relative projectivity}

In this appendix, following Broué [3], we shall state briefly the theory on projectivity relative to bimodules over symmetric algebras. We also add some facts.

Let $A, B$ and $C$ be symmetric $k$-algebras of finite dimension. We let denote by ${ }_{A} \bmod _{C}$ the category of finitely generated $(A, C)$-bimodules whose left and right $k$-module structures coincide. For $L, L^{\prime} \in{ }_{A} \bmod _{C}$ we shall denote by ${ }_{A}\left(L, L^{\prime}\right)_{C}$ the set of $(A, C)$-homomorphisms from $L$ to $L^{\prime}$. Similarly, the symbol ${ }_{A}\left(L, L^{\prime}\right)$ means the set of $A$-homomorphisms from the left $A$-module ${ }_{A} L$ to the left $A$-module ${ }_{A} L^{\prime}$.

Let $X$ be an $(A, B)$-bimodule such that the left $A$-module ${ }_{A} X$ and the right $B$-module $X_{B}$ are both finitely generated and projecive. Then the functors

$$
\begin{aligned}
& { }_{X} S:{ }_{B} \bmod _{C} \rightarrow{ }_{A} \bmod _{C} ; M \mapsto X \otimes_{B} M, \\
& { }_{X} T:{ }_{A} \bmod _{C} \rightarrow{ }_{B} \bmod _{C} ; L \mapsto X^{*} \otimes_{A} L
\end{aligned}
$$

are biadjoint pair. Namely, for arbitrary $M \in{ }_{B} \bmod _{C}$ and $L \in{ }_{A} \bmod _{C}$, we have natural transformations

$$
\begin{aligned}
& \varphi_{L, M}:{ }_{A}\left(X \otimes_{B} M, L\right)_{C} \stackrel{\sim}{\rightarrow}{ }_{B}\left(M, X^{*} \otimes_{A} L\right)_{C} \\
& \psi_{M, L}:{ }_{B}\left(X^{*} \otimes_{A} L, M\right)_{C} \stackrel{\sim}{\rightarrow}{ }_{A}\left(L, X \otimes_{B} M\right)_{C} .
\end{aligned}
$$

We should notice that the natural transformations above depend on the choice of symmetrizing forms of the algebras $A$ and $B$.

We define $\eta_{X^{*}, X}: B \rightarrow X^{*} \otimes_{A} X$ and $\varepsilon_{X^{*}, X}: X^{*} \otimes_{A} X \rightarrow B$ by the following:

$$
\begin{aligned}
\varphi_{X, B}:{ }_{A}(X, X)_{B} & \stackrel{\sim}{\rightarrow}\left(B, X^{*} \otimes_{A} X\right)_{B} \\
\operatorname{Id}_{X} & \mapsto \eta_{X^{*}, X}, \\
\psi_{B, X}:_{B}\left(X^{*} \otimes_{A} X, B\right)_{B} & \stackrel{\sim}{\rightarrow}(X, X)_{B} \\
\varepsilon_{X^{*}, X} & \mapsto \operatorname{Id}_{X} .
\end{aligned}
$$


Similary, considering the $k$-dual $X^{*}$, we define

$$
\begin{aligned}
\varphi_{X^{*}, A}:{ }_{B}\left(X^{*}, X^{*}\right)_{A} & \stackrel{\simeq}{\rightarrow}\left(A, X \otimes_{B} X^{*}\right)_{A} \\
\operatorname{Id}_{X^{*}} & \mapsto \eta_{X, X^{*}}, \\
\psi_{A, X^{*}}:{ }_{A}\left(X \otimes_{B} X^{*}, A\right)_{A} & \stackrel{\simeq}{\rightarrow}\left(X^{*}, X^{*}\right)_{A} \\
\varepsilon_{X, X^{*}} & \mapsto \operatorname{Id}_{X^{*}} .
\end{aligned}
$$

The maps $\eta_{X^{*}, X}$ and $\varepsilon_{X, X^{*}}$ represent the unit $\operatorname{Id}_{B} \bmod _{C} \rightarrow{ }_{X} T \circ{ }_{X} S$ and the counit ${ }_{X} S \circ{ }_{X} T \rightarrow \operatorname{Id}_{A} \bmod _{C}$ of an adjunction for the adjoint pair $\left({ }_{X} S,{ }_{X} T\right)$; the maps $\eta_{X, X^{*}}$ and $\varepsilon_{X^{*}, X}$ represent the unit $\operatorname{Id}_{A} \bmod _{C} \rightarrow{ }_{X} S \circ{ }_{X} T$ and the counit ${ }_{X} T \circ{ }_{X} S \rightarrow \operatorname{Id}_{B} \bmod _{C}$ of an adjunction for the adjoint pair $\left({ }_{X} T,{ }_{X} S\right)$. Hereafter we shall denote by $\eta_{X}, \eta_{X^{*}}, \varepsilon_{X^{*}}$ and $\varepsilon_{X}$ the maps $\eta_{X^{*}, X}, \eta_{X, X^{*}}, \varepsilon_{X^{*}, X}$ and $\varepsilon_{X, X^{*}}$, respectively.

For the $(A, B)$-bimodule $X$ the relative trace map, which we would like to denote by ${ }^{X} \operatorname{Tr}$, is defined as follows: for $L, L^{\prime} \in{ }_{A} \bmod _{C}$

$$
{ }^{X} \operatorname{Tr}:{ }_{B}\left(X^{*} \otimes_{A} L, X^{*} \otimes_{A} L^{\prime}\right)_{C} \rightarrow{ }_{A}\left(L, L^{\prime}\right)_{C} ; \alpha \mapsto \varepsilon_{X} \circ\left(\operatorname{Id}_{X} \otimes \alpha\right) \circ \eta_{X^{*}}
$$

Proposition 5.1 We have for homomorphisms $\beta: X^{*} \otimes_{A} L \rightarrow X^{*} \otimes_{A} L^{\prime}$, $\alpha: L_{1} \rightarrow L$ and $\alpha^{\prime}: L^{\prime} \rightarrow L_{1}^{\prime}$ that

$$
\alpha^{\prime} \circ{ }^{X} \operatorname{Tr}(\beta) \circ \alpha={ }^{X} \operatorname{Tr}\left(\left(\operatorname{Id}_{X^{*}} \otimes \alpha^{\prime}\right) \circ \beta \circ\left(\operatorname{Id}_{X^{*}} \otimes \alpha\right)\right) .
$$

Proposition 5.2 Let $X$ be an $(A, B)$-bimodule and assume that ${ }_{A} X$ and $X_{B}$ are finitely generated and projective.

(i) Let $Y$ be a $(B, C)$-bimodule and assume that ${ }_{B} Y$ and $Y_{C}$ are finitely generated and projective. Then we obtain ${ }^{X \otimes_{B} Y} \operatorname{Tr}={ }^{X} \operatorname{Tr} \circ{ }^{Y} \operatorname{Tr}$.

(ii) Let $X^{\prime}$ be an $(A, B)$-bimodule and assume that ${ }_{A} X^{\prime}$ and $X^{\prime}{ }_{B}$ are finitely generated and projective. Then we have $\operatorname{Im}{ }^{X \oplus X^{\prime}} \operatorname{Tr}=\operatorname{Im}{ }^{X} \operatorname{Tr}+\operatorname{Im}^{X^{\prime}} \operatorname{Tr}$.

Theorem 5.3 Let $X$ be an $(A, B)$-bimodule such that ${ }_{A} X$ and $X_{B}$ are finitely generated and projecive. For $L \in{ }_{A} \bmod _{C}$ the followings are equivalent to each other.

(i) There exist $(A, C)$-homomorphisms $i: L \rightarrow X \otimes_{B} X^{*} \otimes_{A} L$ and $q: X \otimes_{B}$ $X^{*} \otimes_{A} L \rightarrow L$ such that $q \circ i=\operatorname{Id}_{L}$.

(ii) There exists a module $M \in{ }_{B} \bmod _{C}$ and $(A, C)$-homomorphisms $i: L \rightarrow$ $X \otimes_{B} M$ and $q: X \otimes_{B} M \rightarrow L$ such that $q \circ i=\operatorname{Id}_{L}$.

(iii) There exists a $(B, C)$-homomorphism $\beta: X^{*} \otimes_{A} L \rightarrow X^{*} \otimes_{A} L$ such that ${ }^{X} \operatorname{Tr}(\beta)=\operatorname{Id}_{L}$. 
(iv) There exist an $(A, C)$-homomorphism $q: X \otimes_{B} X^{*} \otimes_{A} L \rightarrow L$ such that $q \circ\left(\eta_{X^{*}} \otimes \operatorname{Id}_{L}\right)=\operatorname{Id}_{L}$.

(v) There exist an $(A, C)$-homomorphism $i: L \rightarrow X \otimes_{B} X^{*} \otimes_{A} L$ such that $\left(\varepsilon_{X^{*}} \otimes \operatorname{Id}_{L}\right) \circ i=\operatorname{Id}_{L}$.

(v') (relative projectivity of $L$ ) For $(A, C)$-homomorphisms $\alpha: L \rightarrow L^{\prime}$ and $\pi: L^{\prime \prime} \rightarrow L^{\prime}$, if there exists a $(B, C)$-homomorphism $\beta: X^{*} \otimes_{A} L^{\prime} \rightarrow$ $X^{*} \otimes_{A} L^{\prime \prime}$ such that $\left(\operatorname{Id}_{X^{*}} \otimes \pi\right) \circ \beta=\operatorname{Id}_{X^{*} \otimes_{A} L^{\prime}}$, then there exists an $(A, C)-$ homomorphism $\widehat{\alpha}: L \rightarrow L^{\prime \prime}$ such that $\pi \circ \widehat{\alpha}=\alpha$.

(iv') (relative injectivity of $L$ ) For $(A, C)$-homomorphisms $\alpha: L^{\prime} \rightarrow L$ and $\iota: L^{\prime} \rightarrow L^{\prime \prime}$, if there exists a $(B, C)$-homomorphism $\beta: X^{*} \otimes_{A} L^{\prime \prime} \rightarrow$ $X^{*} \otimes_{A} L^{\prime}$ such that $\beta \circ\left(\operatorname{Id}_{X^{*}} \otimes \iota\right)=\operatorname{Id}_{X^{*} \otimes_{A} L^{\prime}}$, then there exists an $(A, C)$ homomorphism $\widehat{\alpha}: L^{\prime \prime} \rightarrow L$ such that $\widehat{\alpha} \circ \iota=\alpha$.

A module $L \in{ }_{A} \bmod _{C}$ satisfying one (and then all) of the conditions in Theorem 5.3 is said to be relatively ${ }_{X} S$-projective or simply relatively $X$ projective.

A homomorphism $\alpha: L \rightarrow L^{\prime}$, where $L, L^{\prime} \in{ }_{A} \bmod _{C}$ is said to be relatively ${ }_{X} S$-projective or simply relatively $X$-projective if it factors through $X \otimes_{B} T$ for some $T \in{ }_{B} \bmod _{C}$. Theorem 5.3 implies that a homomorphism $\alpha: L \rightarrow L^{\prime}$ is relatively $X$-projective if and only if it is the image under the relative trace map ${ }^{X} \operatorname{Tr}:{ }_{B}\left(X^{*} \otimes_{A} L, X^{*} \otimes_{A} L^{\prime}\right)_{C} \rightarrow{ }_{A}\left(L, L^{\prime}\right)_{C}$.

Remark. Although in [3] a relatively $X$-projective module is said to be ${ }_{X} S$-split and relatively $X$-projective homomorphism is said to be ${ }_{X} S$-split, we would like to use the term "relatively projective".

By definition we have

$$
{ }^{X} \operatorname{Tr}\left(\operatorname{Id}_{X^{*}}\right)=\varepsilon_{X} \circ \eta_{X^{*}}: A \rightarrow A,
$$

which we denote by $\widehat{\pi}_{X}$. The homomorphism $\widehat{\pi}_{X}$ is relatively $X$-projective.

The element $\pi_{X}=\widehat{\pi}_{X}\left(1_{A}\right) \in Z(A)$ is called a relatively $X$-projecive element and the element $\pi_{X^{*}}=\widehat{\pi}_{X^{*}}\left(1_{B}\right) \in Z(B)$ is called a relatively $X^{*}$-projective element. The map $\widehat{\pi}_{X}: A \rightarrow A$ is given by multiplication by the element $\pi_{X} \in Z(A)$ and the map $\widehat{\pi}_{X^{*}}: B \rightarrow B$ is given by multiplication by the element $\pi_{X^{*}} \in Z(B)$.

Theorem 5.4 Let $X$ be an $(A, B)$-bimodule and assume that ${ }_{A} X$ and $X_{B}$ are finitely generated and projective. Let $Y$ be a $(B, C)$-bimodule and assume that ${ }_{B} Y$ and $Y_{C}$ are finitely generated and projective. Assume that an $(A, C)$ bimodule $L$ is isomorphic to a direct summand of the tensor product $X \otimes_{B} Y$. Then the modules ${ }_{A} L$ and $L_{C}$ are finitely generated and projective, respectively, and the following hold. 
(i) The module L is relatively $X$-projective and the homomorphism $\widehat{\pi}_{L}: A \rightarrow$ $A$ is relatively $X$-projective.

(ii) The module $L^{*}$ is relatively $Y^{*}$-projective and the homomorphism $\widehat{\pi}_{L^{*}}$ : $C \rightarrow C$ is relatively $Y^{*}$-projective.

Proof. By definition the module $L$ is relatively $X$-projective and the homomorphism $\widehat{\pi}_{L}$ factors through $X \otimes_{B} Y \otimes L^{*}$. Taking duals we have the assertion (ii).

By Proposition 5.1 we see

Proposition 5.5 Let $X$ be an $(A, B)$-bimodule and assume that ${ }_{A} X$ and $X_{B}$ are finitely generated and projective. For modules $L, L^{\prime} \in{ }_{A} \bmod _{C}$ we let

$$
X^{*} \operatorname{Res}:{ }_{A}\left(L, L^{\prime}\right)_{C} \rightarrow{ }_{B}\left(X^{*} \otimes_{A} L, X^{*} \otimes_{A} L^{\prime}\right)_{C} ; f \mapsto \operatorname{Id}_{X^{*}} \otimes f .
$$

Then we have

$$
{ }^{X} \operatorname{Tr} \circ X^{*} \operatorname{Res}=\widehat{\pi}_{X}\left(=\text { multiplication by } \pi_{X}\right) .
$$

Theorem 5.6 Let $X$ be an $(A, B)$-bimodule and assume that ${ }_{A} X$ and $X_{B}$ are finitely generated and projective. If the relatively projective element $\pi_{X} \in$ $Z(A)$ is invertible, then arbitrary $L \in{ }_{A} \bmod _{C}$ is relatively $X$-projective.

Proof. The assumption that $\pi_{X} \in Z(A)$ is invertible implies that the relative trace map ${ }^{X} \operatorname{Tr}:{ }_{B}\left(X^{*} \otimes_{A} L, X^{*} \otimes_{A} L\right)_{C} \rightarrow{ }_{A}(L, L)_{C}$ is epimoriphic because of the previous proposition.

\section{References}

1. Alperin, J. L., M. Linckelmann, and R. Rouquier: 2001, 'Source algebras and source modules'. J. Algebra 239(1), 262-271.

2. Benson, D. J. and M. Linckelmann: 2005, 'Vertex and source determine the block variety of an indecomposable module'. J. Pure Appl. Algebra 197, 11-17.

3. Broué, M.: 1991, On representations of symmetric algebras: an introduction, Notes by M. Stricker. Mathematik Department ETH Zürich.

4. Kawai, H. and H. Sasaki: 2006, 'Cohomology algebras of blocks of finite groups and Brauer correspondence'. Algebr. Represent. Theory 9(5), 497-511.

5. Kessar, R., M. Linckelmann, and G. R. Robinson: 2002, 'Local control in fusion systems of p-blocks of finite groups'. J. Algebra 257(2), 393-413.

6. Linckelmann, M.: 1999a, 'Transfer in Hochschild cohomology of blocks of finite groups'. Algebr. Represent. Theory 2, 107-135.

7. Linckelmann, M.: 1999b, 'Varieties in block theory'. J. Algebra 215, 460-480.

8. Nagao, H. and Y. Tsushima: 1989, Representations of Finite Groups. New York, London: Academic Press. 